\title{
Interferometric Measurement of Co-Operative Evaporation in 2-D Droplet Arrays
}

\author{
Andrew M. J. Edwards * ${ }^{1}$ Jack Cater, ${ }^{1}$ Joseph J. Kilbride, ${ }^{1}$ Pierre Le Minter, ${ }^{1}$ Carl V. Brown, ${ }^{1}$ David J. \\ Fairhurst, ${ }^{1,}$ a) and Fouzia F. Ouali ${ }^{1, b)}$ \\ SOFT Group, School of Science and Technology, Nottingham Trent University, Clifton Lane, Nottingham NG11 8NS, \\ $U K$.
}

(Dated: 16 November 2021)

\begin{abstract}
The evaporation dynamics of multiple droplet arrays is important in inkjet printing and spray coating, where cooperative "shielding" effects increase overall evaporation time. However, current measurement methods provide limited information on how individual droplets contribute to the overall dynamics. In this work, we present a simple interferometric technique for precise measurements of droplet evaporation rates which is not possible via traditional approaches. We validate the technique on a single droplet. We then extend our analysis to ordered and random 2-D arrays of droplets. We demonstrate that the evaporation rate is highly dependent on the position of the droplet within the array and its confinement. The more confined droplets exhibit lower evaporation rates. Our results of 10 different configurations and well over 100 droplets are in good quantitative agreement with a recent analytical model of co-operative evaporation rates. Our approach opens up possibilities for studies of collective evaporation effects, including in areas of current importance such as sneezes and exhaled breath.
\end{abstract}

The evaporation of a liquid droplet on a solid surface is of importance in a wide range of industrial applications such as coating and printing ${ }^{1,2}$. The main body of research into this problem has focused on single evaporating droplets on solid surfaces ${ }^{2-4}$. More recently, the focus of evaporation research has shifted to studying multiple droplets which are relevant in deposited sprays $\mathrm{s}^{5,6}$, splash patterns $^{7}$, inkjet printing ${ }^{8}$ and in influencing viral transmission through droplets generated when coughing and sneezing ${ }^{9-12}$. In these situations, the presence of neighbouring droplets increases the local vapour concentration such that the evaporation rate of an individual droplet depends on all the surrounding droplets, often referred to as a "shielding" effect ${ }^{13}$. This reduced evaporation rate has been shown to change the internal flow structure ${ }^{14,15}$, influence the shape of final deposits ${ }^{16-19}$, and result in striking final morphologies ${ }^{20}$. Interestingly, other work has demonstrated that evaporating (or dissolving) droplet arrays can induce convection in the vapour phase which can lead to droplets evaporating faster in an array than when isolated ${ }^{6,8}$. A similar observation is also seen for micron sized droplets ${ }^{21,22}$, here due to the dependence of vapour pressure on the droplet curvature via the Kelvin effect.

The recent surge in theoretical investigations of collective evaporation of multiple droplets and sprays calls for experimental validation of the resulting models and simulations. For isolated evaporating droplets, measurements are typically made using a camera to capture magnified images of the droplet profile. Temporal measurements of the height $h$, radius $r$, and contact angle $\theta$, are extracted from the images which allows the determination of evaporation rate. Whilst the profile imaging method works well for multiple droplets arranged in a line ${ }^{15}$, it is unable to image each droplet simultaneously within a 2-D array as some droplets will inevitably be obscured by others. This limits the measurement

\footnotetext{
${ }^{a)}$ Electronic mail: Corresponding Author: david.fairhurst@ntu.ac.uk

b)Electronic mail: Corresponding Author: fouzia.ouali@ntu.ac.uk
}

of multiple droplet arrays to top-down imaging and gravimetric analysis 6,23 , which provide limited information on individual droplet evaporation time and the collective evaporation rate respectively. Interferometry has previously been used to measure profiles of low contact angle droplets ${ }^{24,25}$, distortions due to Marangoni effects ${ }^{26}$, and to image the local vapour concentration and temperature profile around evaporating droplets ${ }^{25,27}$.

In this work, we develop a time dependent, spatially resolved interferometric technique to measure the evaporation dynamics of pinned droplets on surfaces irrespective of their contact angle. We confirm the validity of the technique by comparing to traditional imaging methods on a single droplet, showing excellent agreement between the two methods. We then apply our technique to measure individual droplet evaporation rates in 10 organised and random 2-D droplet arrays, demonstrating how individual evaporation rate is dependent on position within the array and proximity to neighbouring droplets. We also demonstrate good agreement with recent predictions. $^{28}$

To perform the interferometric measurement, we use a Mach-Zehnder interferometer with a He-Ne polarised laser (HRP170, ThorLabs) with a peak wavelength, $\lambda=632.8 \mathrm{~nm}$, as the light source. We use a spatial filter system to obtain a Gaussian intensity profile. The expanded beam is passed through a beam splitter, creating a 'reference' and 'sample' beam. The divided beams are recombined at the second beam splitter where interference patterns are created due to differences in optical path length between the two paths. To image our samples, we use a relay lens system which allows magnified images of the droplets to be captured by the camera ${ }^{29}$.

We have developed a simple and flexible method for selectively hydrophobising patterned substrates so that each droplet's contact line remains pinned for most of the evaporation. Labelling tape is adhered to a clean hydrophilic glass slide having an arithmetic mean deviation surface roughness, $R a=0.6 \pm 0.3 \mathrm{~nm}$. The templated circular incisions are laser cut through the tape which is then carefully removed leav- 

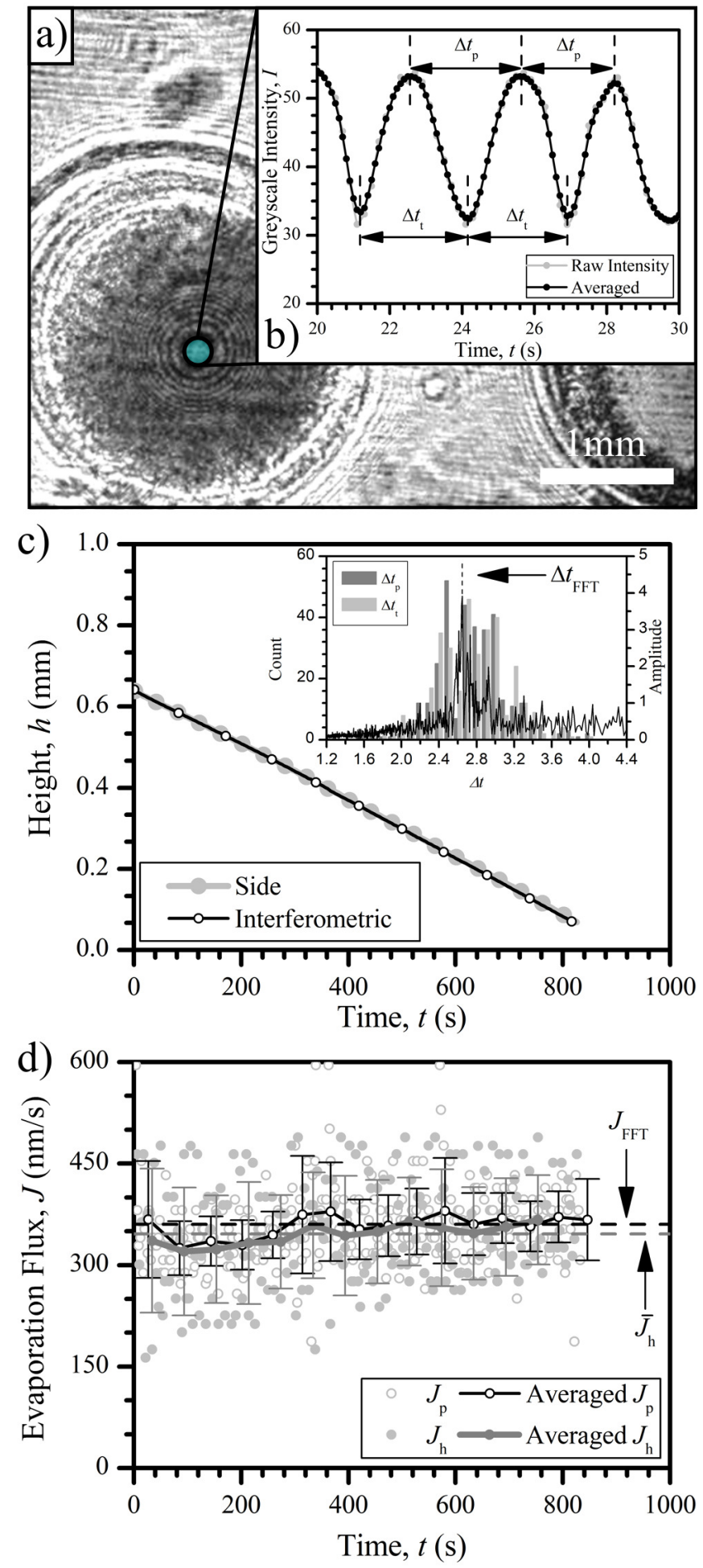

FIG. 1. a) Experimental image of evaporating droplet. Blue spot shows the circular area over which greyscale intensity is measured. b) Greyscale intensity of the spot with time. c) Height of the evaporating droplet with time determined from side and interferometric measurement. Inset shows histogram of $\Delta t_{\mathrm{p}}$ and $\Delta t_{\mathrm{t}}$ and FFT power spectrum (solid line) for experimental data. d) Temporal measurement of average evaporation flux obtained from both interferometric and side measurements for the droplet shown in Fig. 1a. ing the tape covering the circular dots only. The slide is then coated with the hydrophobic coating NOVEC2702 with $R a=39.5 \pm 0.8 \mathrm{~nm}^{30}$. Removal of the templates exposes patterned hydrophilic regions on a hydrophobic surface. We find that deposited droplets spread to fill the hydrophilic regions and remain pinned for around $80 \%$ of the total evaporation time. Substrates are placed in a controlled environment with ambient temperature, $T=20 \pm 1^{\circ} \mathrm{C}$ and relative humidity, $R H=43 \pm 6 \%$. We use deionsied water, obtained from a PURELAB Chorus 1 (ELGA) with surface tension, $\gamma=72.75 \mathrm{mN} / \mathrm{m}$, density, $\rho=998.21 \mathrm{~kg} / \mathrm{m}^{3}$ and refractive index, $n=1.33211 @ 632.8 \mathrm{~nm}^{31}$. We deposit droplets of volume $V=1.0 \pm 0.1 \mu \mathrm{L}$ on to the hydrophilic regions for which the total deposition time is less than $2 \%$ of the overall evaporation time. The contact angles of deposited droplets are $\theta=$ $64 \pm 4^{\circ}$. The Bond number, $\rho g h^{2} / \gamma \approx 10^{-2}$ indicates that the droplets can be considered spherically capped.

When a droplet is placed in the sample arm of the interferometer, interference fringes appear due to the difference in optical path length through different parts of the curved droplet ${ }^{24}$ (Fig. 1a). For a pinned evaporating droplet with any contact angle value, the height of the droplet decreases over time ${ }^{32}$, shortening the optical path length and changing the resultant interference intensity across the droplet ${ }^{25}$. Fig. $1 \mathrm{~b}$ shows the mean greyscale value within the indicated circular area, with a radius of $86 \mu \mathrm{m}$, at the apex of the droplet as a function of time, after applying a moving average filter to minimise noise (black line in Fig. 1b). By measuring the time separation between peaks $\Delta t_{p}$ and between troughs $\Delta t_{t}$, we are able to determine the time taken to reduce the optical path by one wavelength, corresponding to a decrease in droplet height of $\Delta h_{f}=\lambda /(n-1)=1.905 \mu \mathrm{m}^{25}$.

We validate our approach using simultaneous interferometric and profile imaging on a single droplet, providing a comparison of the accuracy of the measured change in height and therefore the measured evaporation flux between the two methods. The results of this comparison are shown in Fig. 1c, where, to determine the absolute height of the droplet as a function of time for the interferometer data, we start with the initial measured height from the profile image and iteratively reduce the height by $\Delta h_{f}$ after each fringe. We find excellent quantitative agreement between the two techniques.

The volume of a spherical cap, $V$ is given by Eq. $1^{33}$.

$$
V=\frac{\pi}{6}\left(3 r^{2} h+h^{3}\right)
$$

Differentiating Eq. 1 gives the change in volume resulting from a height change for a pinned droplet as

$$
\frac{d V}{d t}=\frac{\pi}{2}\left(r^{2}+h^{2}\right) \frac{d h}{d t}
$$

The average evaporation flux through a surface is given by $J=(d V / d t) / A$, where the total surface area is given by $A=$ $\pi\left(r^{2}+h^{2}\right)$ for a spherical cap. Therefore, we can determine the average temporal evaporation flux using $J=(d h / d t) / 2$. For our profile measurements, the flux, denoted $J_{\mathrm{h}}$, can be found directly from the slope of the droplet height against 


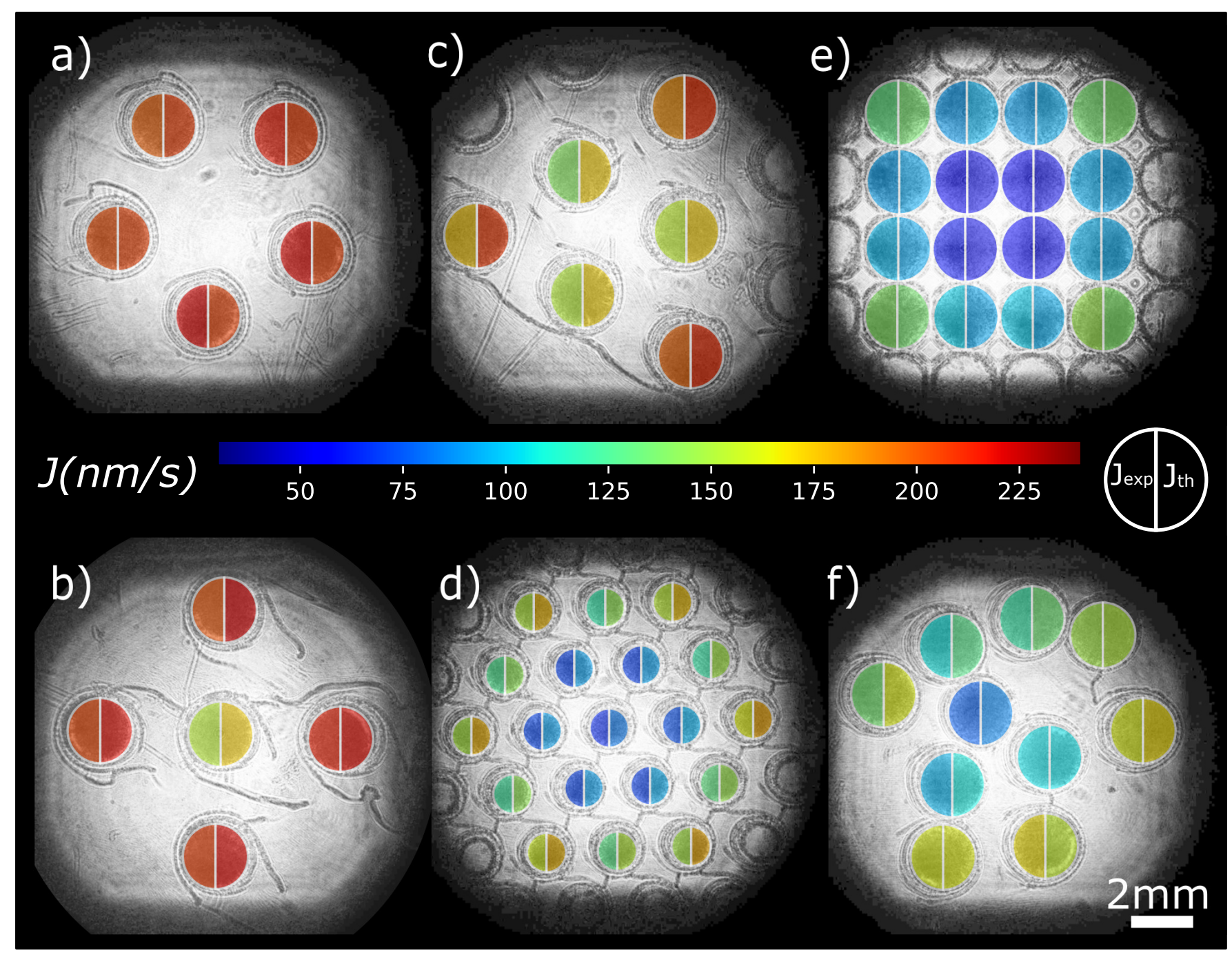

FIG. 2. Experimental images of evaporating 2-D droplet arrays. Colored overlay shows; measured average evaporation flux (left side), $J_{\text {exp }}$ and Wray et al's theoretically predicted evaporation flux (right side), $J_{\text {th }}$, in (nm/s), for between $50 \mathrm{~s}$ to $250 \mathrm{~s}$ after final droplet deposition. a) Pentagon. b) Cross. c) Triangle. d) Hexagon. e) Square. f) Random. Images have been histogram equalised.

time (Fig. 1c). For the interferometric technique, we can measure the evaporation flux using three approaches: the times between peaks $\Delta t_{\mathrm{p}}$, the times between troughs $\Delta t_{\mathrm{t}}$, and by taking a fast Fourier transform (FFT) of the intensity data. We note that the peak-to-peak and trough-to-trough measurements provide the instantaneous evaporation flux throughout the evaporation, while the FFT approach provides the average flux for the whole dataset by finding the peak of the power spectrum which corresponds to the dominant period of oscillation, $\Delta t_{\mathrm{FFT}}$. The inset in Fig. 1c shows the histogram of the measured $\Delta t_{\mathrm{p}}, \Delta t_{\mathrm{t}}$ and FFT power spectrum. We quantify the error on our measurements of the peak and trough methods using the standard deviation of the distribution of measured $\Delta t_{\mathrm{p}}$ and $\Delta t_{\mathrm{t}}$, and for the FFT approach using the full width at half maximum of the peak of the power spectrum.

Fig. 1d shows the droplet evaporation flux as a function of time for both profile and interferometric methods showing very similar average values and a similar level of noise. We find the average values for evaporation flux of the interferometric method $\left(\bar{J}_{\mathrm{p}} \approx 360 \pm 60 \mathrm{~nm} / \mathrm{s}, \bar{J}_{\mathrm{t}} \approx 360 \pm 60 \mathrm{~nm} / \mathrm{s}\right.$ and $J_{\mathrm{FFT}} \approx 360 \pm 10 \mathrm{~nm} / \mathrm{s}$ ) are comparable to the droplet profile method $\left(\bar{J}_{\mathrm{h}} \approx 350 \pm 80 \mathrm{~nm} / \mathrm{s}\right)$. The interferometric method has a lower uncertainty due to the higher precision in determining the incremental change in droplet height.

Next we apply the interferometric technique to the case of multiple identical interacting droplets in 2-D arrays, both ordered and random, as shown in Fig. 2. For simplicity, we combine our interferometric measurements using $J_{\exp }=$ $\left(\bar{J}_{\mathrm{p}}+\bar{J}_{\mathrm{t}}+J_{\mathrm{FFT}}\right) / 3$, and use this measured flux to colour the left half of each droplet in Fig. 2. As expected, the effect of shielding on a droplet, and therefore its evaporation flux, is determined by its position within the array, number of neighbouring droplets and their proximity (which we term the droplet's "confinement"). Droplets in equivalent/symmetric positions have the same confinement, and so experience identical shielding effects from neighbouring droplets and evapo- 
rate at the same rate, while those at the corners are less confined, so evaporate faster, than those at the edges, and those in the centre evaporate most slowly.

Fig. 2a shows the measured evaporation flux for droplets arranged in a pentagon where the average evaporation flux varies by no more than $7 \%$ between droplets. This follows from the symmetry of the pentagon providing identical confinement conditions for each droplet. In comparison to an isolated droplet, co-operative shielding reduces the flux of each droplet by $\approx 62 \%$. Rearranging the five droplets into a cross formation (Fig. 2b) creates two confinement conditions: the points and the centre. The points of the cross being least confined have the highest evaporation flux (a reduction of only $33 \pm 5 \%$ ) while the evaporation flux of the centre droplet has reduced by $50 \%$. The triangle formation in Fig. 2c illustrates that droplets at the corners of the triangle (two nearest neighbours) are less confined than those on the edges (four nearest neighbours), with the former evaporating approximately $20 \%$ faster than the latter.

The dense droplet array configurations in Fig. 2d, e again show that the evaporation flux is highest from the least confined droplets at the corners and decreases with increasing confinement. Interestingly, within the filled hexagon (Fig. 2d) we find that the six inner ring droplets evaporate around $50 \%$ slower than the outer droplets; however, the centre droplet evaporates only $8 \%$ slower than the inner ring. This suggests that the influence of the edge of the pattern only extends two droplets into the array; further inwards the evaporation rate is constant. Fig. $2 \mathrm{f}$ shows a random arrangement of evaporating droplets where each droplet has a unique confinement, similar to a spray pattern.

We compare our experimental results to the predictions of Wray et al. ${ }^{28}$. They used an asymptotic approach of a model of potential flow through porous membranes, first developed by Fabrikant ${ }^{34}$, to calculate how the evaporative flux of the $k^{\text {th }}$ droplet $\left(J_{k}\right)$ in an array of $N$ well-separated droplets with low contact angle is reduced compared to its flux when evaporating in isolation $\left(J_{0_{k}}\right)$.

$$
J_{k}=J_{0_{k}}-\frac{2}{\pi} \sum_{\substack{n=1 \\ n \neq k}}^{N}\left(\frac{r_{n}}{r_{k}}\right)^{2} J_{n} \arcsin \left(\frac{r_{k}}{d_{k n}}\right)
$$

Here, $r_{k / n}$ is the base radius of droplet $k / n$, and $d_{k n}$ is the distance between droplets $k$ and $n$. The model assumes the droplets interact only via diffusion in the vapour phase. Equation (3) is the same equation as that derived by Fabrikant ${ }^{34}$.

To apply this result, we start by rearranging and writing Equation (3) in matrix form $\Phi \mathbf{J}=\mathbf{J}_{\mathbf{0}}$ where $\Phi$ is an $N \times N$ matrix with off diagonal elements that capture the interaction between droplets $k$ and $n$. For droplets of equal radius $r_{k}=r_{n}$, off-diagonal elements in this matrix are given by $\phi_{k n}=\frac{2}{\pi} \arcsin \left(\frac{r_{k}}{d_{k n}}\right)$ and diagonal elements $\phi_{n n}=1$. By inverting $\Phi$ we can solve for the matrix of unknown droplet fluxes $\mathbf{J}$ (with elements $J_{k}$ ) in terms of the corresponding matrix of known isolated droplet fluxes $\mathbf{J}_{\mathbf{0}}$ (with elements $J_{0_{k}}$ ) such that $\mathbf{J}=\Phi^{-1} \mathbf{J}_{\mathbf{o}}$ (see Supplementary Material for more details). A single isolated flux value $J_{0_{k}}=J_{0}$ of a droplet with known radius $r_{0}$ can be used in place of the matrix $\mathbf{J}_{\mathbf{0}}$ provided this value is scaled by ratio of $r_{0}$ to $r_{k}$ i.e. $\mathbf{J}=\Phi^{-1}\left(r_{o} / r_{k}\right) J_{o}$. Contributions to the uncertainties in the calculated elements of $\Phi$ are around $10 \%$ from $r_{k}$ and $1 \%$ from $d_{k n}$. Treating these as systematic errors leads to around a $4 \%$ error in the elements of $\Phi^{-1}$, whereas if the measurement errors are randomly distributed, the effect on $\Phi^{-1}$ is only around $0.5 \%$. Therefore, errors in determining $J_{k}$ mainly arise from errors associated with measuring $J_{0_{k}}=J_{0}$.

We use the same independently measured value for $J_{0_{k}}=J_{0}$ for droplets with the same size as they would have the same flux if evaporating in isolation. There are no adjustable parameters. We use these calculated fluxes to colour code the right side of each droplet in Fig. 2. The pentagon (a) and square (e) show excellent agreement (to within 5\%) and the other four configurations (to within $20 \%$ for (d)) also confirm that the spatial dependence of evaporation flux on position within the 2-D array is well captured by the model. The largest difference in the hexagon (d) may be attributed to small variations in droplet size within the smaller diameter droplet array.

To quantify this comparison fully, in Fig. 3 we plot our experimental measurements of evaporation flux against the theoretical flux calculated from Wray et al. ${ }^{28}$ for droplets in a larger number of configurations (see Fig. S1 in Supplementary Material), including error bars on all points. The solid line is a linear fit to the data, including the error margin, with a gradient of $1.1 \pm 0.2$ which shows a good agreement with the model. Wray et al. note that their asymptotic approach provides surprising agreement despite only being formally valid for flat, well-separated droplets. Here we also find a good agreement for high contact angle droplets, some of which are almost touching. The good agreement with the diffusive theory suggests that additional effects, such as convection ${ }^{8}$ or thermal interactions, do not appear to be experimentally significant although we do not rule out that that they may account for some of the deviations at high evaporation flux. More experiments are needed to confirm this fully.

In this work, we have demonstrated how the evaporative flux of a pinned droplet can be measured using a MachZehnder interferometer, with higher accuracy than standard profile imaging. The technique is applicable to pinned droplets in 2-D arrays irrespective of their contact angle. This includes droplets with high contact angles, which act as high numerical aperture lenses, and thus are challenging to image using conventional profiling techniques. We applied this technique to both ordered and random 2-D arrays, extracting the simultaneous evaporation fluxes of all individual droplets. We have been able to quantify the different shielding conditions within 2-D arrays, demonstrating very good agreement with the analytical model of Wray et al. ${ }^{28}$, and thus providing the first rigorous experimental validation of a theoretical model of co-operative evaporation effects. In our arrays, the dynamics are dominated by diffusion, with no evidence of convection or thermal interactions. For other liquids and substrates, our approach would allow direct observation and quantitative measurements of these collective effects, which could provide insights into the dynamics of co-operative evaporation in multiple droplet arrays relevant to cooling ${ }^{35}$, printing $^{36}$, coating ${ }^{37}$, 


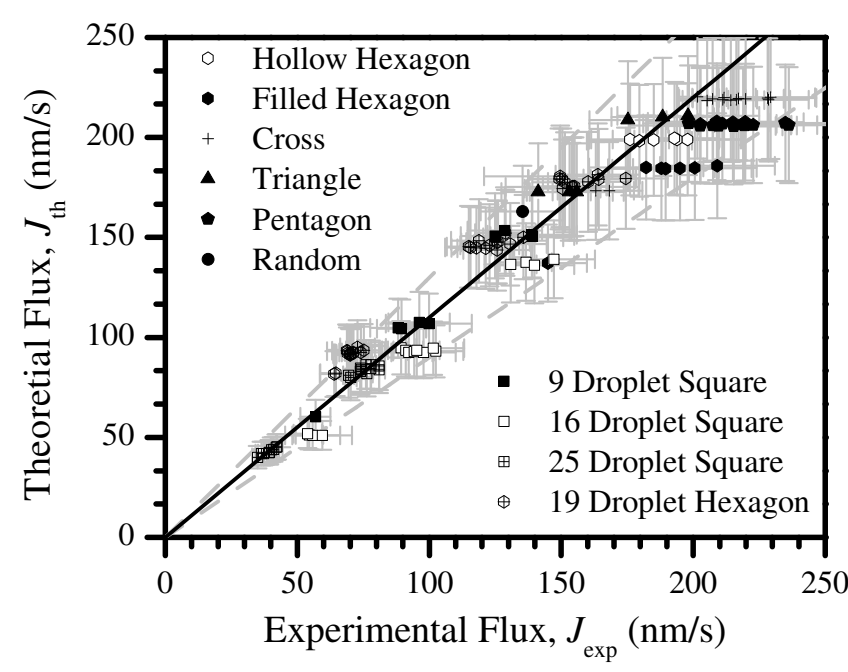

FIG. 3. Comparison between experimentally measured evaporation flux of 10 arrays and theoretical flux calculated using the analytical model of Wray et al. ${ }^{28}$, for all droplets in Fig. 2 and other arrangements (see Fig. S1 in the Supplementary Material). The solid line is a linear fit of slope $1.1 \pm 0.2$ with the dashed lines showing the uncertainty limits.

DNA arrays ${ }^{38}$, and important current areas such as sneezes and breath figures ${ }^{39}$.

See Supplementary Material for a complete explanation of the matrix approach and for complete series of overlaid experimental images (Fig. S1). The data that support the findings of this study are available from the corresponding author upon reasonable request.

\section{ACKNOWLEDGEMENT}

We would like to thank G. J. Hickman for atomic force microscopy measurements of surface roughness.

\section{* Now at Loughborough University.}

${ }^{1}$ J. Sun, B. Bao, M. He, H. Zhou, and Y. Song, "Recent advances in controlling the depositing morphologies of inkjet droplets," ACS Applied Materials \& Interfaces 7, 28086-28099 (2015).

${ }^{2}$ D. Brutin, Droplet wetting and evaporation: from pure to complex fluids (Academic Press, 2015).

${ }^{3}$ R. D. Deegan, O. Bakajin, T. F. Dupont, G. Huber, S. R. Nagel, and T. A. Witten, "Capillary flow as the cause of ring stains from dried liquid drops," Nature 389, 827-829 (1997).

${ }^{4}$ R. G. Larson, "Transport and deposition patterns in drying sessile droplets," AIChE Journal 60, 1538-1571 (2014).

${ }^{5}$ S. Kooij, R. Sijs, M. M. Denn, E. Villermaux, and D. Bonn, "What determines the drop size in sprays?" Physical Review X 8, 031019 (2018).

${ }^{6}$ O. Carrier, N. Shahidzadeh-Bonn, R. Zargar, M. Aytouna, M. Habibi, J. Eggers, and D. Bonn, "Evaporation of water: evaporation rate and collective effects," Journal of Fluid Mechanics 798, 774-786 (2016).

${ }^{7}$ A. L. Yarin, "Drop impact dynamics: splashing, spreading, receding, bouncing. ..," Annu. Rev. Fluid Mech. 38, 159-192 (2006).

${ }^{8}$ G. Laghezza, E. Dietrich, J. M. Yeomans, R. Ledesma-Aguilar, E. S. Kooij, H. J. Zandvliet, and D. Lohse, "Collective and convective effects com- pete in patterns of dissolving surface droplets," Soft Matter 12, 5787-5796 (2016).

${ }^{9}$ C. Y. H. Chao, M. Wan, and G. Sze To, "Transport and removal of expiratory droplets in hospital ward environment," Aerosol Science and Technology 42, 377-394 (2008).

${ }^{10}$ R. Bhardwaj and A. Agrawal, "How coronavirus survives for days on surfaces," Physics of Fluids 32, 111706 (2020).

${ }^{11}$ C. S. Ng, K. L. Chong, R. Yang, M. Li, R. Verzicco, and D. Lohse, "Growth of respiratory droplets in cold and humid air," Physical Review Fluids 6, 054303 (2021).

${ }^{12}$ K. L. Chong, C. S. Ng, N. Hori, R. Yang, R. Verzicco, and D. Lohse, "Extended lifetime of respiratory droplets in a turbulent vapor puff and its implications on airborne disease transmission," Physical Review Letters 126, 034502 (2021).

${ }^{13}$ F. G. Schofield, A. W. Wray, D. Pritchard, and S. K. Wilson, "The shielding effect extends the lifetimes of two-dimensional sessile droplets," Journal of Engineering Mathematics 120, 89-110 (2020).

${ }^{14}$ T. K. Pradhan and P. K. Panigrahi, "Influence of an adjacent droplet on fluid convection inside an evaporating droplet of binary mixture," Colloids and Surfaces A: Physicochemical and Engineering Aspects 500, 154-165 (2016).

${ }^{15}$ S. Hatte, K. Pandey, K. Pandey, S. Chakraborty, and S. Basu, "Universal evaporation dynamics of ordered arrays of sessile droplets," Journal of Fluid Mechanics 866, 61-81 (2019).

${ }^{16}$ R. D. Deegan, O. Bakajin, T. F. Dupont, G. Huber, S. R. Nagel, and T. A. Witten, "Contact line deposits in an evaporating drop," Physical Review E 62, 756 (2000).

${ }^{17}$ T. K. Pradhan and P. K. Panigrahi, "Deposition pattern of interacting droplets," Colloids and Surfaces A: Physicochemical and Engineering Aspects 482, 562-567 (2015).

${ }^{18} \mathrm{~S}$. Hu, Y. Wang, X. Man, and M. Doi, "Deposition patterns of two neighboring droplets: Onsager variational principle studies," Langmuir 33, 59655972 (2017).

${ }^{19}$ A. J. D. Shaikeea and S. Basu, "Evaporating sessile droplet pair: Insights into contact line motion, flow transitions and emergence of universal vaporisation pattern," Applied Physics Letters 108, 244102 (2016).

${ }^{20}$ L. Chen and J. R. Evans, "Arched structures created by colloidal droplets as they dry," Langmuir 25, 11299-11301 (2009).

${ }^{21}$ A. Lacasta, I. Sokolov, J. Sancho, and F. Sagués, "Competitive evaporation in arrays of droplets," Physical Review E 57, 6198 (1998).

${ }^{22}$ C. Schäfle, C. Bechinger, B. Rinn, C. David, and P. Leiderer, "Cooperative evaporation in ordered arrays of volatile droplets," Physical Review Letters 83, 5302 (1999).

${ }^{23}$ K. Pandey, S. Hatte, K. Pandey, S. Chakraborty, and S. Basu, "Cooperative evaporation in two-dimensional droplet arrays," Physical Review E 101, 043101 (2020).

${ }^{24}$ R. Fondecave and F. B. Wyart, "Polymers as dewetting agents," Macromolecules 31, 9305-9315 (1998).

${ }^{25}$ S. Dehaeck, I. Tsoumpas, and P. Colinet, "Measuring 3d droplet shape and local slope using interferometry and continuous wavelet transforms," in 10th Pacific Symposium on Flow Visualization and Image Processing (2015).

${ }^{26}$ Y. Tsoumpas, S. Dehaeck, A. Rednikov, and P. Colinet, "Effect of marangoni flows on the shape of thin sessile droplets evaporating into air," Langmuir 31, 13334-13340 (2015).

${ }^{27}$ F. Braig, F. Narrog, H. M. Sauer, and E. Dörsam, "Interferometric imaging of solvent vapor of evaporating liquid films," Langmuir (2021).

${ }^{28}$ A. W. Wray, B. R. Duffy, and S. K. Wilson, "Competitive evaporation of multiple sessile droplets," Journal of Fluid Mechanics 884 (2020).

${ }^{29}$ C. Brown, G. Wells, M. Newton, and G. McHale, "Voltage-programmable liquid optical interface," Nature Photonics 3, 403-405 (2009).

${ }^{30}$ A. M. J. Edwards, R. Ledesma-Aguilar, M. I. Newton, C. V. Brown, and G. McHale, "A viscous switch for liquid-liquid dewetting," Communications Physics 3, 1-6 (2020).

${ }^{31}$ D. Lide, CRC Handbook of Chemistry and Physics (Boca Raton, 2003).

${ }^{32} \mathrm{H}$. Y. Erbil, "Evaporation of pure liquid sessile and spherical suspended drops: A review," Advances in Colloid and Interface Science 170, 67-86 (2012).

${ }^{33} \mathrm{~W}$. Kern and J. Bland, Solid mensuration with proofs 2 nd Ed. (Wiley, New York, 1938). 
${ }^{34}$ V. Fabrikant, "On the potential flow through membranes," Zeitschrift für angewandte Mathematik und Physik ZAMP 36, 616-623 (1985).

${ }^{35}$ T. Kokalj, H. Cho, M. Jenko, and L. Lee, "Biologically inspired porous cooling membrane using arrayed-droplets evaporation," Applied Physics Letters 96, 163703 (2010).

${ }^{36} \mathrm{P}$. Calvert, "Inkjet printing for materials and devices," Chemistry of Materials 13, 3299-3305 (2001).
${ }^{37}$ J. Pyeon and H. Kim, "Controlling uniform patterns by evaporation of multi-component liquid droplets in a confined geometry," Soft Matter 17, 3578-3585 (2021).

${ }^{38}$ V. Dugas, J. Broutin, and E. Souteyrand, "Droplet evaporation study applied to dna chip manufacturing," Langmuir 21, 9130-9136 (2005).

${ }^{39}$ J. Rodríguez-Hernández and E. Bormashenko, Breath figures: Mechanisms of Multi-Scale Patterning and Strategies for Fabrication and Applications of Microstructured Functional Porous Surfaces (Springer Nature, 2020). 